Research Article

\title{
A New Conflict Management in Evidence Theory Based on DEMATEL Method
}

\author{
Wentao Fan $(1)$ and Fuyuan Xiao \\ School of Computer and Information Science, Southwest University, Chongqing 400715, China \\ Correspondence should be addressed to Fuyuan Xiao; doctorxiaofy@hotmail.com
}

Received 22 July 2019; Revised 10 October 2019; Accepted 31 October 2019; Published 19 November 2019

Academic Editor: Jesús Lozano

Copyright (c) 2019 Wentao Fan and Fuyuan Xiao. This is an open access article distributed under the Creative Commons Attribution License, which permits unrestricted use, distribution, and reproduction in any medium, provided the original work is properly cited.

D-S evidence theory is widely used in data fusion. However, the result of Dempster's combination rule is not efficient and in highly conflicting situation. Though the existing methods have been proved efficient to deal with conflict in some applications, the indirect conflict among evidence is neglected to some degree. To solve this problem, a new method is proposed based on decision-making trial and evaluation laboratory (DEMATEL) and the belief correlation coefficient in this paper. The application in target recognition illustrates the efficiency of the proposed method. Compared with Dempster's rule, averaging method and weighted averaging method, etc., the results obtained by the proposed method have better performance. The main reason is that the indirect conflict is well addressed in the proposed method.

\section{Introduction}

Uncertainty information processing is an open issue in many fields [1-3]. Except for probability theory, many methods have been proposed to deal with uncertainty: exploited fuzzy sets $[4,5], D$-numbers [6-9], $Z$-numbers $[10,11], R$-numbers [12], evidential reasoning [13-16], fluid structure analysis [17, 18], entropy [19-21], evidence theory [22], and so on [23]. As one of the useful methods, D-S evidence theory has the advantage to deal with the uncertainty in a flexible and reasonable way [24], so that it is widely used in many applications, including uncertainty measurement in decision-making [25-27], information fusion [28], target recognition $[29,30]$, diagnosis [31, 32], and risk analysis [33].

However, conflict management [34-36] in evidence theory is not well addressed. This limitation greatly decreases efficiency of evidence theory in a highly conflicting situation [37]. In [38], a method called evidence distance proposed by Jousselme et al. is proposed to measure the conflict degree among evidence. Liu [39] found that the classical conflict coefficient $k$ cannot effectively measure the degree of conflict between two pieces of evidence. Therefore, a two-dimensional conflict model which takes the pignistic probability distance and the conflict coefficient $k$ together to represent the degree of conflict is presented. Song et al. [40] used the positive fixed matrix $D$ to preprocess the BPAs and measure the conflicts between the evidences bodies. Yager [41] proposed the application of dependency as a discounting factor for multievidence fusion. Murphy [42] proposed the application of averaging for multiple evidence fusion. Based on Murphy's method and evidence distance, Deng et al. [43] considered the weight of evidence and proposed the weight averaging method. However, these methods can only show direct conflict between two pieces of evidence, where the indirect relationship among evidence is not considered $[44,45]$. Indirect conflict exists when there are three or more BPAs. Assume there are three BPAs $A, B, C$, there is not only conflict between BPA $A$ and BPA $B$ and conflict between evidence BPA $B$ and BPA $C$ but also the transitive influence between BPA $A$ and BPA $C$. The transitive influence is called indirect conflict.

Recently, a new correlation coefficient of belief functions is effective for measuring the conflict of two pieces of evidence [46]. To address indirect conflict management, a new method to combine belief function based on DEMATEL 
(decision-making trial and evaluation laboratory) [47, 48] and the correlation coefficient is proposed in this paper. DEMATEL method is a system factor analysis method based on graph theory and matrix proposed by the Bottele Institute in the United States which has been applied into evaluationmaking, decision-making, and so on. Recently, a new method based on DEMATEL and single-valued neutrosophic numbers proposed by Liu et al. [49] shows advantage in multicriteria decision-making. Another advantage of DEMATEL is that it fully takes into account the indirect effects between elements. In the steps of DEMATEL method, normalized matrix continues to multiply and then add the results together, this process is to superimpose the indirect effects of the elements. So if the direct conflict of evidence is taken as an element of DEMATEL, the indirect conflict among evidence can be modeled with DEMATEL.

The paper is organized as follows. Section 2 is the brief introduction of the preliminaries, reviewing the classic D-S evidence theory, a new correlation coefficient to measure the conflict among evidence proposed by Jiang, the averaging method, and DEMATEL method. In Section 3, the new correlation coefficient is applied to DEMATEL method to obtain each evidence's discounting factor. In Section 4, some numerical examples are shown to combine the conflicting evidence by the proposed method and the final result is compared with the results obtained by different methods. In Section 5, a short discussion about the advantage compared with Dempster's rule, averaging method, weighted averaging method, and Song et al.'s method is presented. In Section 6, a brief conclusion is presented.

\section{Preliminaries}

In this section, some preliminaries, including D-S evidence theory, conflict management, correlation coefficients of BPA, and DEMATEL method, are briefly introduced.

2.1. D-S Evidence Theory. Dealing with uncertainty is inevitable in real world [50-52]. Among the existing math tools [53-55], evidence theory is widely used due to its efficiency $[56,57]$. The identification framework is the most basic concept in evidence theory, originating from what people can know and what they want to know [58]. Any concern proposition corresponds to a subset of the identification framework $\theta$. If the following equation is true

$$
m(\phi)=0, \sum_{A \subseteq \theta} m(A)=1
$$

then $m: 2^{\theta} \longrightarrow[0,1]$ is called the basic probability assignment (BPA) $[59,60]$ on $\theta$, also known as the mass function. $2^{\theta}$ denotes a power set of $\theta$, containing all subsets of $\theta$. Many relative operations on BPA are presented such as negation [61], divergence [62], and entropy function [63].

Set the identification framework to $\theta$ and suppose there are $N$-independent BPAs, $m_{1}, m_{2}, \cdots, m_{N}$, which can be fused into one BPA by the combination rule of $\mathrm{D}-\mathrm{S}$ evidence theory. The combination rule is shown as follows:

$$
m(A)= \begin{cases}0 & \text { if } A=\phi \\ K^{1} \sum_{\cap A_{j}=A} \prod_{i=1}^{n} m_{i}\left(A_{j}\right) & \text { otherwise }\end{cases}
$$

where

$$
K=1-\sum_{\cap A_{j}=\theta} \prod_{i=1}^{n} m_{i}\left(A_{j}\right) .
$$

When discounting combination is performed, $\alpha$ is assumed to be the weight of one evidence, and $m_{i}$ is one of the BPAs based on the identification framework $\theta$, and then the discounted mass function can be expressed as follows:

$$
m_{i}^{\prime}(A)= \begin{cases}a m(A) & \text { if } A \subset \theta, A \neq \theta, \\ 1-a+a m(\theta) & \text { otherwise. }\end{cases}
$$

2.2. Conflict Management. Sometimes the conflict among two BPAs will make the result obtained by Dempster's combination rule unreasonable $[64,65]$. Suppose that $\theta$ is a frame of discernment with three elements $A, B, C$. Assume two BPAs, from two distinct sources, are defined as follows:

$$
\begin{aligned}
& m_{1}(A)=0.9, m_{1}(B)=0.1, m_{1}(C)=0.0 \\
& m_{2}(A)=0.0, m_{2}(B)=0.1, m_{2}(C)=0.9
\end{aligned}
$$

Combining $m_{1}$ and $m_{2}$ using Dempster's combination rule leads to a new BPA $m_{3}$ with $m_{3}(B)=1.0$ and $m_{3}(\phi)=0.99$ before normalization. Neither of the two strongly preferred choices by the two sources is preserved and the least preferred choice by the two sources is given the full credit after the combination with Dempster's combination rule.

In order to address this issue, Murphy [42] proposed the averaging method. Suppose there is a total of $N$ BPAs, Murphy proposed that if all evidence is in an identification framework, the quality can be averaged first and then combined for $N-1$ times using Dempster's combination rule.

However, in reality, the importance of each evidence is different, so the weight of each evidence needs to be determined before the fusion. Deng et al. apply the distance of evidence proposed by Jousselme et al. [66] to calculate the weight of each evidence. Assume that the identification framework is $\theta$, and regard all subsets of $\theta$ as a vector. A BPA is a vector $m \sim$ of the vector space. The distance is shown as follows [66]:

$$
d\left(m_{1}, m_{2}\right)=\left(\vec{m}_{1}-\vec{m}_{2}\right)^{\mathrm{T}} \underline{\underline{D}}\left(\vec{m}_{1}-\vec{m}_{2}\right) .
$$

$\underline{D}$ is a $2^{N} \times 2^{N}$ matrix. Let $D(A, B)$ be the coefficient of the matrix, where

$$
D(A, B)=\left|\frac{A \cap B}{A \cup B}\right|
$$

Distance function can be used to measure the difference of two sets [67]. The distance of evidence is applied to 
calculate the degree of association between one and the other; then, the degree of association of each evidence is used as the weight in multiple evidence fusion. We can use Dempster's combination rule to combine the weighted average of the masses for $N-1$ times to get the final result. For more detailed information, refer [43].

2.3. Correlation Coefficients for Belief Functions. The methods to measure the conflict between two BPAs have been presented by some researchers [68]. The association coefficient $\operatorname{cor}\left(m_{1}, m_{2}\right)$ obtained by Song et al. [40] uses the positive definite matrix $D$ to preprocess the two BPAs, $m_{1}^{\prime}=m_{1} D$ and $m_{2}^{\prime}=m_{2} D, D$ is a positive fixed matrix as in Equation (6).

$$
\operatorname{cor}\left(m_{1}, m_{2}\right)=\frac{\left\langle m_{1}^{\prime}, m_{2}^{\prime}\right\rangle}{\left\|m_{1}^{\prime}\right\| \cdot\left\|m_{2}^{\prime}\right\|}
$$

where $\left\langle m_{1}^{\prime}, m_{2}^{\prime}\right\rangle$ is the inner product of vectors, and $\left\|m_{1}^{\prime}\right\|$ is the norm of vector.

Recently, a new correlation coefficient of belief functions is proposed by Jiang [46] which is effective for measuring the conflict of two pieces of evidence. The coefficient is under the conditions of considering nonintersection and the difference among the focal elements.

Assuming the identification framework is $\theta$ and $m_{1}, m_{2}$, $\cdots, m_{n}$ are $N$ BPAs, the correlation between $m_{1}$ and $m_{2}$ can be represented as follows [46]:

$$
r_{\mathrm{BPA}}\left(m_{1}, m_{2}\right)=\frac{c\left(m_{1}, m_{2}\right)}{\sqrt{c\left(m_{1}, m_{1}\right) c\left(m_{2}, m_{2}\right)}},
$$

where

$$
c\left(m_{1}, m_{2}\right)=\sum_{i=1}^{2^{N}} \sum_{j=1}^{2^{N}} m_{1}\left(A_{i}\right) m_{2}\left(A_{j}\right)\left|\frac{A_{i} \cap A_{j}}{A_{i} \cup A_{j}}\right|,
$$

where $i, j=1,2 \cdots 2^{N} ; A_{i}, A_{j}$ is the focal elements of mass, respectively; $|\cdot|$ is the cardinality of a subset.

And the conflict between $m_{1}$ and $m_{2}$ is denoted as follows [46]:

$$
c_{\mathrm{BPA}}\left(m_{1}, m_{2}\right)=1-r_{\mathrm{BPA}}\left(m_{1}, m_{2}\right)
$$

2.4. DEMATEL Method. Many methods such as complex network [69, 70] and bioinspired model [71-74] have be applied to deal with the complexity in the real world. DEMATEL method [47] is a system factor analysis method based on graph theory and matrix proposed by the Bottele Institute in the United States. The basic idea of DEMATEL is to use matrix operations to calculate the system component's degree of influence and degree of being influenced to obtain each component's importance and casual relationship and then use the importance and casual relationship to adjust the structure of the original system which makes the decision-making more reasonable.
Step 1. Determine the influencing factors of the system and generate the direct relation matrix.

The major steps are as follows [75]:

$$
D=\left[\begin{array}{cccc}
0 & a_{12} & \cdots & a_{1 n} \\
a_{21} & 0 & \cdots & a_{2 n} \\
\vdots & \vdots & \ddots & \vdots \\
a_{n 1} & a_{n 2} & \cdots & 0
\end{array}\right],
$$

where $a_{i j}$ denotes the influence between the two factors $i$ and $j$

Step 2. Normalize the direct relation matrix.

The normalized direct relation matrix $N$ is obtained as follows [75]:

$$
N_{n \times n}=S \times D_{n \times n}
$$

where

$$
S=\frac{1}{\max \left[\sum_{j=1}^{n} a_{i j}, \sum_{i=1}^{n} a_{i j}\right]} .
$$

Each element of the normalized direct relation matrix $N$ falls between zero and one.

Step 3. Calculate total relation matrix.

Total relation matrix $T$ is obtained as follows [75]:

$$
T=\lim _{n \rightarrow \infty}\left(N+N^{1}+N^{2}+\cdots+N^{n}\right)=N(I-N)^{-1},
$$

where $I$ is the identity matrix.

Step 4. Calculate the causal parameters $R$ and $C$.

$R$ refers to the sum of each row and $C$ refers to the sum of each column as shown below [75]:

$$
\begin{gathered}
R_{i}=\sum_{j} T_{i j}(i=1,2, \cdots, n), \\
C_{j}=\sum_{i} T_{i j}(i=1,2, \cdots, n) .
\end{gathered}
$$

Step 5. Calculate the prominence of each criterion. $\beta$ denotes the prominence of each criterion which shows the influence of each criterion and the degree of being influenced. It is calculated from the following formula [75]:

$$
\beta=R_{i}+C_{i}
$$

\section{Proposed Method}

For better conflict management and a more realistic fusion result, it is necessary to take the indirect conflict among 
evidence in the system into consideration. In this section, a new conflict management method based on DEMATEL is proposed. The flowchart of the proposed algorithm is illustrated in Figure 1.

Suppose that $N$ pieces of evidence are obtained from different sensors, which can be expressed as $S_{1}, S_{2}, S_{3}, \cdots, S_{N}$. Given two evidence $S_{i}$ and $S_{j}$, the direct correlation denotes as $R_{s i, s j}$, and the direct conflict denotes as $C_{s i, s j}$. In this paper, the proposed method takes the conflict among BPAs calculated by the correlation coefficient as the influencing factor of the system and generates the direct relation matrix.

Given the correlation coefficient $R_{s i, s j}$, the corresponding correlation coefficient matrix $R$ is defined as follows:

$$
R=\left[\begin{array}{cccc}
R_{S_{1}, S_{1}} & R_{S_{1}, S_{2}} & \cdots & R_{S_{1}, S_{N}} \\
R_{S_{2}, S_{1}} & R_{S_{2}, S_{2}} & \cdots & R_{S_{2}, S_{N}} \\
\vdots & \vdots & \ddots & \vdots \\
R_{S_{N}, S_{1}} & R_{S_{N}, S_{2}} & \cdots & R_{S_{N}, S_{N}}
\end{array}\right] .
$$

Given the direct conflict $C_{s i, s j}$, the direct relation matrix $D$ is defined as follows:

$$
D=\left[\begin{array}{cccc}
C_{S_{1}, S_{1}} & C_{S_{1}, S_{2}} & \cdots & C_{S_{1}, S_{N}} \\
C_{S_{2}, S_{1}} & C_{S_{2}, S_{2}} & \cdots & C_{S_{2}, S_{N}} \\
\vdots & \vdots & \ddots & \vdots \\
C_{S_{N}, S_{1}} & C_{S_{N}, S_{2}} & \cdots & C_{S_{N}, S_{N}}
\end{array}\right],
$$

where

$$
C_{S_{i}, S_{j}}=1-R_{S_{i}, S_{j}}
$$

In order to analyze the indirect relationship among factors, it is necessary to normalize the direct relation matrix. The direct relation matrix $D$ is normalized in Equations (13) and (14).

Given the direct relation matrix $D$, the normalized matrix $M$ is defined as follows:

$$
M=\left[\begin{array}{cccc}
C_{S_{1}, S_{1}}^{\prime} & C_{S_{1}, S_{2}}^{\prime} & \cdots & C_{S_{1}, S_{N}}^{\prime} \\
C_{S_{2}, S_{1}}^{\prime} & C_{S_{2}, S_{2}}^{\prime} & \cdots & C_{S_{2}, S_{N}}^{\prime} \\
\vdots & \vdots & \ddots & \vdots \\
C_{S_{N}, S_{1}}^{\prime} & C_{S_{N}, S_{2}}^{\prime} & \cdots & C_{S_{N}, S_{N}}^{\prime}
\end{array}\right],
$$

where

$$
C_{S_{i}, S_{j}}^{\prime}=\frac{C_{S_{i}, S_{j}}}{\max \left(\sum_{j=1}^{n} C_{S_{i}, S_{j}}, \sum_{i=1}^{n} C_{S_{i}, S_{j}}\right)} .
$$

Normalized matrix continues to multiply and then add, this process is to superimpose the indirect effects of

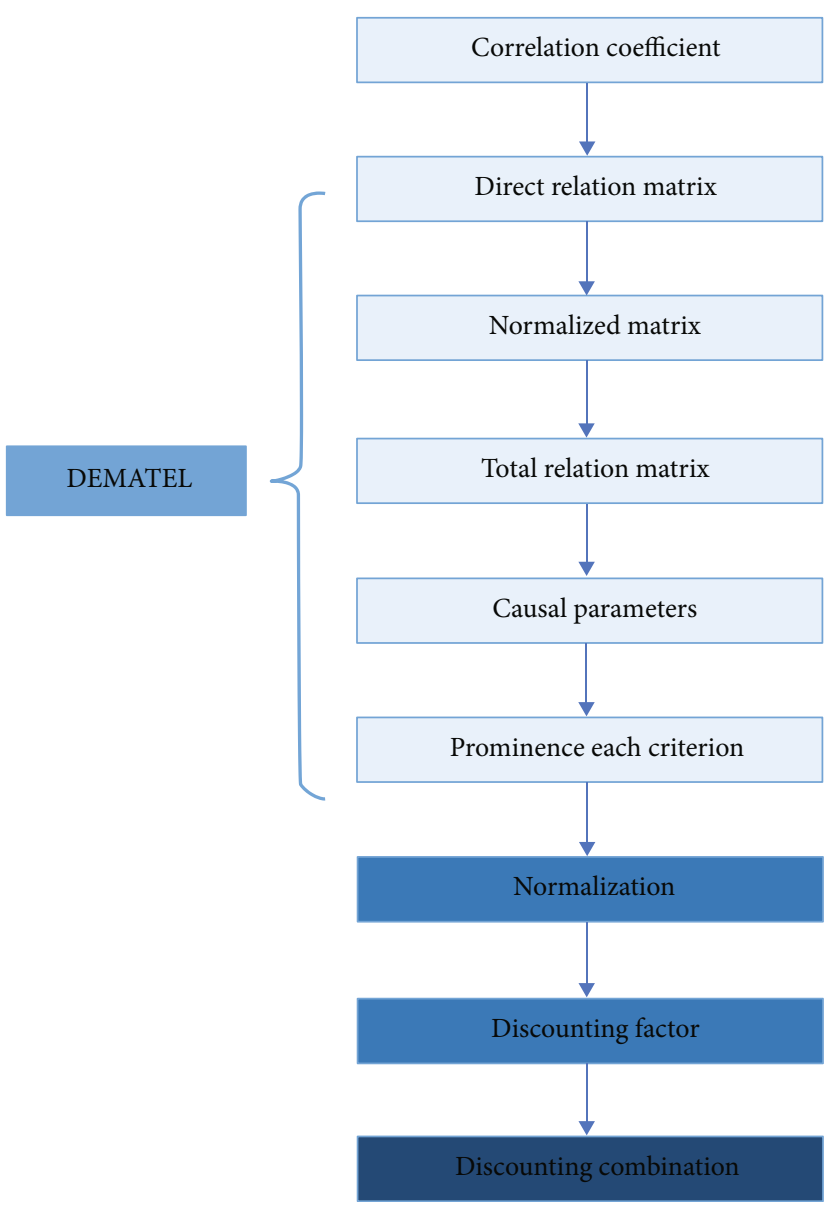

FIGURE 1: The algorithm of the proposed method.

the elements. The total relation matrix $T$ is calculated in Equation (15).

Given the normalized matrix $M$, the total relation matrix $T$ is defined as follows:

$$
T=\left[\begin{array}{cccc}
C_{S_{1}, S_{1}}^{\prime \prime} & C_{S_{1}, S_{2}}^{\prime \prime} & \cdots & C_{S_{1}, S_{N}}^{\prime \prime} \\
C_{S_{2}, S_{1}}^{\prime \prime} & C_{S_{2}, S_{2}}^{\prime \prime} & \cdots & C_{S_{2}, S_{N}}^{\prime \prime} \\
\vdots & \vdots & \ddots & \vdots \\
C_{S_{N}, S_{1}}^{\prime \prime} & C_{S_{N}, S_{2}}^{\prime \prime} & \cdots & C_{S_{N}, S_{N}}^{\prime \prime}
\end{array}\right]
$$

where

$$
\begin{aligned}
C_{S_{i}, S_{j}}^{\prime \prime} & =\lim _{n \rightarrow \infty}\left(C_{S_{i}, S_{j}}+\left(C_{S_{i}, S_{j}}^{\prime}\right)^{2}+\cdots+\left(C_{S_{i}, S_{j}}^{\prime}\right)^{n}\right) \\
& =\frac{C_{S_{i}, S_{j}}^{\prime}}{1-C_{S_{i}, S_{j}}^{\prime}} .
\end{aligned}
$$

$R$ refers to the degree of influence, which refers to the combined effect of the element on other elements. $C$ refers to the degree of being influenced, which refers to the total influence of the element being affected by other elements. 
The causal parameters $R$ and $C$ are calculated in Equations (16) and (17).

$$
\left\{\begin{array}{l}
R_{i}=\sum_{j} C_{S_{i}, S_{j}}^{\prime \prime}(i=1,2, \cdots, n), \\
C_{j}=\sum_{i} C_{S_{i}, S_{j}}^{\prime \prime}(i=1,2, \cdots, n) .
\end{array}\right.
$$

The prominence of each criterion is calculated in Equation (18).

$$
\beta=R_{i}+C_{i} .
$$

The prominence of each criterion is used to get the discounting factor.

The discounting factor is defined as follows:

$$
\alpha=\frac{1}{\beta} .
$$

$\alpha$ is applied as discounting factor in the proposed method, in Equation (4). Because the direct conflict of evidence is taken as the influencing factor of the direct relationship matrix, the results obtained by DEMATEL signify the extent to which the BPA affects the entire system in a conflict-based manner. The larger the value obtained, the greater the conflict between the BPA and other BPAs. So the larger the result, the more it proves that this is a bad $\mathrm{BPA}$, and its weight is naturally lower than other BPAs. This is why in this article, the reciprocal of the prominence of each criterion is used as a discounting factor.

The sum of the discounting factors obtained from the above formula may not be exactly equal to 1 , in which case normalization is also required.

The algorithm of the proposed method is shown in Algorithm 1.

\section{Numerical Example}

4.1. Example 1. Assume that in a multisensor system, five sensors obtained five bodies of evidence, respectively. Suppose the real target is $F_{1}$, the five bodies of evidence are shown as follows, where $\theta=\left(F_{1}, F_{2}, F_{3}\right)$ is the frame of discernment:

$$
\begin{aligned}
& m_{1}\left(F_{1}\right)=0.7, m_{1}\left(F_{2}\right)=0.1, m_{1}(\theta)=0.2, \\
& m_{2}\left(F_{1}\right)=0.7, m_{2}(\theta)=0.3, \\
& m_{3}\left(F_{1}\right)=0.65, m_{3}\left(F_{2}\right)=0.15, m_{3}(\theta)=0.2, \\
& m_{4}\left(F_{1}\right)=0.75, m_{4}\left(F_{3}\right)=0.05, m_{4}(\theta)=0.2, \\
& m_{5}\left(F_{2}\right)=0.2, m_{5}\left(F_{3}\right)=0.8 .
\end{aligned}
$$

The steps of this experiment are shown as follows:

Step 1. Obtain the correlation coefficient matrix based on the correlation coefficient of BPA; the results are shown in Table 1.

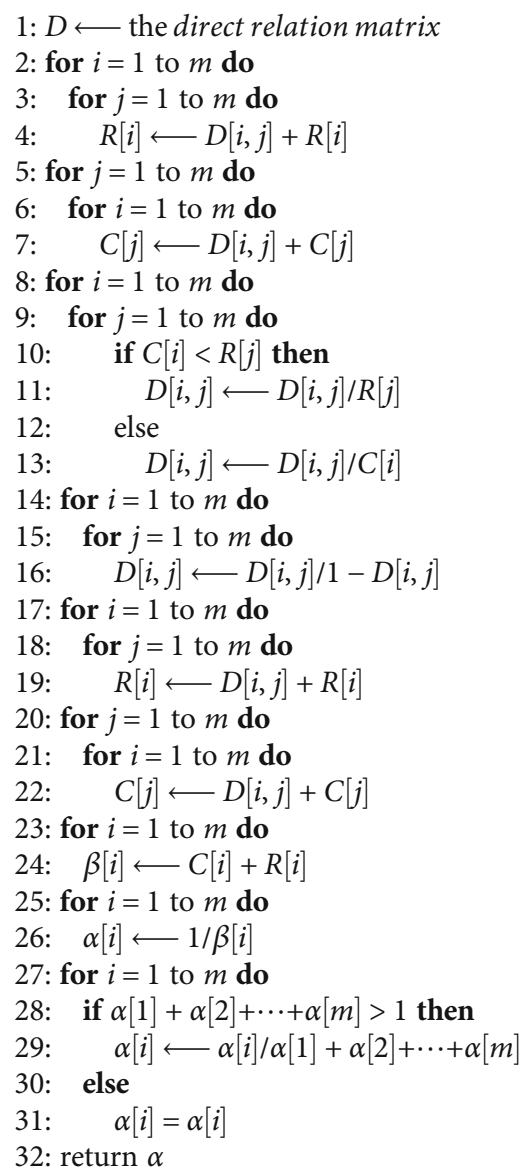

Algorithm 1: New conflict management based on DEMATEL method.

TABLE 1: The correlation coefficient matrix for example 1.

\begin{tabular}{cccccc}
\hline & $m_{1}$ & $m_{2}$ & $m_{3}$ & $m_{4}$ & $m_{5}$ \\
\hline$m_{1}$ & 1 & 0.9918 & 0.9969 & 0.9901 & 0.1307 \\
$m_{2}$ & 0.9918 & 1 & 0.9831 & 0.9943 & 0.1429 \\
$m_{3}$ & 0.9969 & 0.9831 & 1 & 0.9773 & 0.1524 \\
$m_{4}$ & 0.9901 & 0.9943 & 0.9773 & 1 & 0.1534 \\
$m_{5}$ & 0.1307 & 0.1429 & 0.1524 & 0.1534 & 1 \\
\hline
\end{tabular}

Step 2. Based on the correlation coefficient, calculate the conflict among BPAs.

Step 3. Obtain the direct relation matrix based on the conflict among BPAs; the results are shown in Table 2.

Step 4. Obtain the normalized matrix based on the direct relation matrix; the results are shown in Table 3.

Step 5. Obtain the total relation matrix based on the normalized matrix; the results are shown in Table 4. 
TABLE 2: The direct relation matrix for example 1 .

\begin{tabular}{cccccc}
\hline & $m_{1}$ & $m_{2}$ & $m_{3}$ & $m_{4}$ & $m_{5}$ \\
\hline$m_{1}$ & 0 & 0.0082 & 0.0031 & 0.0099 & 0.8639 \\
$m_{2}$ & 0.0082 & 0 & 0.0169 & 0.0057 & 0.8571 \\
$m_{3}$ & 0.0031 & 0.0169 & 0 & 0.0227 & 0.8476 \\
$m_{4}$ & 0.0099 & 0.0057 & 0.0227 & 0 & 0.8466 \\
$m_{5}$ & 0.8639 & 0.8571 & 0.8476 & 0.8466 & 0 \\
\hline
\end{tabular}

TABle 3: The normalized matrix for example 1.

\begin{tabular}{cccccc}
\hline & $m_{1}$ & $m_{2}$ & $m_{3}$ & $m_{4}$ & $m_{5}$ \\
\hline$m_{1}$ & 0 & 0.0092 & 0.0035 & 0.0119 & 0.2530 \\
$m_{2}$ & 0.0092 & 0 & 0.0190 & 0.0064 & 0.2510 \\
$m_{3}$ & 0.0035 & 0.0190 & 0 & 0.0255 & 0.2482 \\
$m_{4}$ & 0.0119 & 0.0064 & 0.0255 & 0 & 0.2479 \\
$m_{5}$ & 0.2530 & 0.2510 & 0.2482 & 0.2479 & 0 \\
\hline
\end{tabular}

TABLe 4: The total relation matrix for example 1.

\begin{tabular}{cccccc}
\hline & $m_{1}$ & $m_{2}$ & $m_{3}$ & $m_{4}$ & $m_{5}$ \\
\hline$m_{1}$ & 0 & 0.0093 & 0.0035 & 0.0120 & 0.3387 \\
$m_{2}$ & 0.0093 & 0 & 0.0194 & 0.0065 & 0.3351 \\
$m_{3}$ & 0.0035 & 0.0194 & 0 & 0.0262 & 0.3301 \\
$m_{4}$ & 0.0120 & 0.0065 & 0.0262 & 0 & 0.3296 \\
$m_{5}$ & 0.3387 & 0.3351 & 0.3301 & 0.3296 & 0 \\
\hline
\end{tabular}

TABLE 5: The causal parameters $R$ for example 1 .

\begin{tabular}{llllll}
\hline$R$ & 0.3635 & 0.3703 & 0.3792 & 0.3743 & 1.3335 \\
\hline
\end{tabular}

TABle 6: The causal parameters $C$ for example 1.

\begin{tabular}{llllll}
\hline$C$ & 0.3635 & 0.3703 & 0.3792 & 0.3743 & 1.3335 \\
\hline
\end{tabular}

TABLE 7: The prominence of each criterion for example 1.

\begin{tabular}{lcccc}
\hline$m_{1}$ & $m_{2}$ & $m_{3}$ & $m_{4}$ & $m_{5}$ \\
\hline 0.727 & 0.7406 & 0.7584 & 0.7486 & 2.6667 \\
\hline
\end{tabular}

Step 6. Calculate the causal parameters $R$ and $C$ based on total relation matrix; the results are shown in Tables 5 and 6 .

Step 7. Use parameters $R$ and $C$ to calculate the prominence of each criterion; the results are shown in Table 7.

Step 8. Use the prominence of each criterion to calculate each evidence's discounting factor; the results are shown in Table 8 .

Step 9. Normalize the discounting factor to get each evidence's weight; the results are shown in Table 9.
TABLE 8: The discounting factor for example 1.

\begin{tabular}{lcccc}
\hline$m_{1}$ & $m_{2}$ & $m_{3}$ & $m_{4}$ & $m_{5}$ \\
\hline 1.3755 & 1.3502 & 1.3186 & 1.3358 & 0.3750 \\
\hline
\end{tabular}

TABLE 9: Normalized discounting factor for example 1.

\begin{tabular}{lcccc}
\hline$m_{1}$ & $m_{2}$ & $m_{3}$ & $m_{4}$ & $m_{5}$ \\
\hline 0.2390 & 0.2346 & 0.2291 & 0.2321 & 0.0652 \\
\hline
\end{tabular}

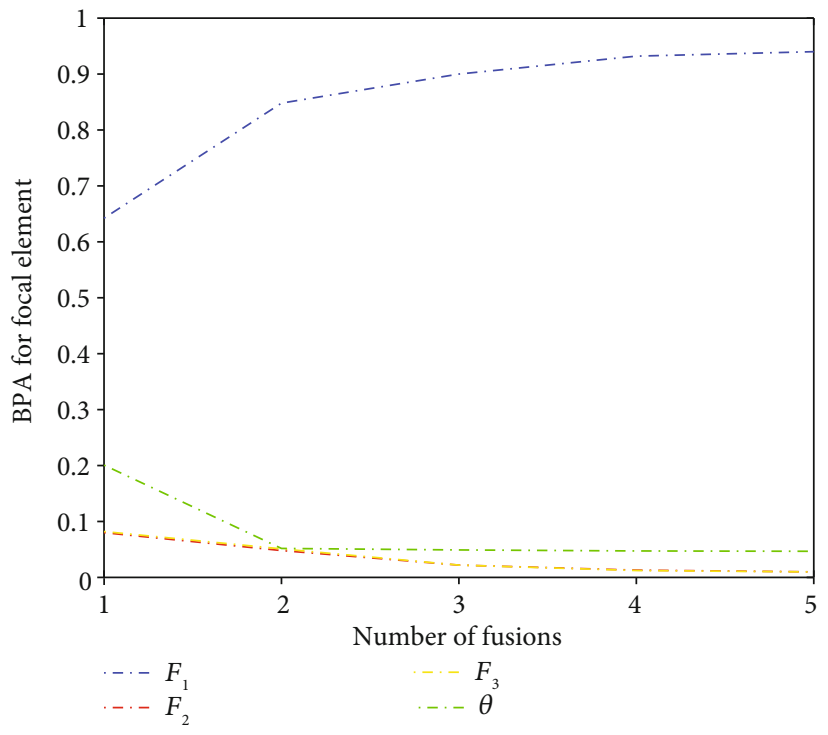

FIGURE 2: BPA for each focal element.

And the probability of each element during the data fusion is shown in Figure 2 in which we can observe that the proposed method provides most support for the real target $F_{1}$.

In order to make example 1 better understand and more convincing, detailed step-by-step calculations for one specific element are presented. We take the element in the first row and the fifth column as example. First calculate the correlation coefficient $r_{\mathrm{BPA}}\left(m_{1}, m_{2}\right)$ according to Equation (9) and Equation (10). By calculation, $c\left(m_{5}, m_{5}\right)$ is equal to $0.68, c\left(m_{1}, m_{1}\right)$ is equal to 0.6466 , so we can get $r_{\mathrm{BPA}}\left(m_{1}, m_{5}\right)=0.1307$, as shown in Table 1 . Then by calculating $c_{\mathrm{BPA}}\left(m_{1}, m_{5}\right)$ by Equation (11), we can get $c_{\text {BPA }}\left(m_{1}, m_{5}\right)=0.8639$ as shown in Table 2 . Then use Equation (23) to normalize the matrix. The sum of the first row is 0.8851, while the sum of the fifth column is 3.4152. So we divide 0.8639 by 3.4152 to get 0.2530 as shown in Table 3.

Then use Equation (25) to get the result 0.3387 as shown in Table 4.

4.2. Example 2. Suppose there are three BPAs with discernment frame $\{A, B, C\}$ as follows:

$$
\begin{aligned}
& m_{1}(A)=0.7, m_{1}(B)=0.1, m_{1}(C)=0.2, \\
& m_{2}(A)=0.6, m_{2}(B)=0.3, m_{2}(C)=0.1, \\
& m_{3}(A)=a, m_{3}(B)=b .
\end{aligned}
$$


TABle 10: Example 2.

\begin{tabular}{lcc}
\hline Value of $a$ & Discounting factor & Mass of $A$ \\
\hline 0 & $0.2182,0.5162,0.2156$ & 0.5191 \\
0.1 & $0.2341,0.5875,0.1784$ & 0.7633 \\
0.2 & $0.2126,0.6159,0.1715$ & 0.8 \\
0.3 & $0.1839,0.6548,0.1613$ & 0.8362 \\
0.4 & $0.1472,0.7173,0.1355$ & 0.8669 \\
0.5 & $0.1033,0.7942,0.1025$ & 0.8854 \\
0.6 & $0.1444,0.6940,0.1616$ & 0.9041 \\
0.7 & $0.2210,0.4603,0.3187$ & 0.9215 \\
0.8 & $0.2763,0.2974,0.4263$ & 0.9756 \\
0.9 & $0.3913,0.2811,0.3276$ & 0.9876 \\
1 & $0.2122,0.5711,0.2167$ & 0.9776 \\
\hline
\end{tabular}

TABle 11: The correlation coefficient matrix for example 3.

\begin{tabular}{cccccc}
\hline & $m_{1}$ & $m_{2}$ & $m_{3}$ & $m_{4}$ & $m_{5}$ \\
\hline$m_{1}$ & 1 & 0.3762 & 0.8908 & 0.8908 & 0.8921 \\
$m_{2}$ & 0.3762 & 1 & 0.1499 & 0.1499 & 0.1449 \\
$m_{3}$ & 0.8908 & 0.1499 & 1 & 1 & 0.9981 \\
$m_{4}$ & 0.8908 & 0.1499 & 1 & 1 & 0.9981 \\
$m_{5}$ & 0.8921 & 0.1449 & 0.9981 & 0.9981 & 1 \\
\hline
\end{tabular}

TABLE 12: The direct relation matrix for example 3.

\begin{tabular}{cccccc}
\hline & $m_{1}$ & $m_{2}$ & $m_{3}$ & $m_{4}$ & $m_{5}$ \\
\hline$m_{1}$ & 0 & 0.6238 & 0.1092 & 0.1092 & 0.1079 \\
$m_{2}$ & 0.6238 & 0 & 0.8501 & 0.8501 & 0.8551 \\
$m_{3}$ & 0.1092 & 0.8501 & 0 & 0 & 0.019 \\
$m_{4}$ & 0.1092 & 0.8501 & 0 & 0 & 0.019 \\
$m_{5}$ & 0.1079 & 0.8551 & 0.019 & 0.019 & 0 \\
\hline
\end{tabular}

TABLE 13: The normalized matrix for example 3.

\begin{tabular}{cccccc}
\hline & $m_{1}$ & $m_{2}$ & $m_{3}$ & $m_{4}$ & $m_{5}$ \\
\hline$m_{1}$ & 0 & 0.1962 & 0.1136 & 0.1136 & 0.1116 \\
$m_{2}$ & 0.1962 & 0 & 0.2674 & 0.2674 & 0.2690 \\
$m_{3}$ & 0.1136 & 0.2674 & 0 & 0 & 0.0020 \\
$m_{4}$ & 0.1136 & 0.2674 & 0 & 0 & 0.0020 \\
$m_{5}$ & 0.1116 & 0.2960 & 0.0020 & 0.0020 & 0 \\
\hline
\end{tabular}

According to the first two BPAs, we can know that the target is $A$. We increase $a$ from 0 to 1 , that is, from a situation which is most contradictory to reality to a situation which is closest to reality, observe the change of mass of $\mathrm{A}$. The results are shown in the Table 10.

Through the above table, even if $a$ is equal to 0 , the result obtained by the proposed method still support target $A$. When $a$ is slightly increased, the mass of $A$ has reached 0.8 or more, which is enough to demonstrate the superiority of this method.
TABLe 14: The total relation matrix for example 3.

\begin{tabular}{cccccc}
\hline & $m_{1}$ & $m_{2}$ & $m_{3}$ & $m_{4}$ & $m_{5}$ \\
\hline$m_{1}$ & 0 & 0.2441 & 0.1282 & 0.1282 & 0.1256 \\
$m_{2}$ & 0.2441 & 0 & 0.3468 & 0.3650 & 0.3680 \\
$m_{3}$ & 0.1282 & 0.3650 & 0 & 0 & 0.0020 \\
$m_{4}$ & 0.1282 & 0.3650 & 0 & 0 & 0.0020 \\
$m_{5}$ & 0.1256 & 0.3680 & 0.0020 & 0.0020 & 0 \\
\hline
\end{tabular}

TABLE 15: The causal parameters $R$ for example 3 .

\begin{tabular}{llllll}
\hline$R$ & 0.6261 & 1.3421 & 0.4952 & 0.4952 & 0.4976 \\
\hline
\end{tabular}

TABLE 16: The causal parameters $C$ for example 3 .

\begin{tabular}{llllll}
\hline$C$ & 0.6261 & 1.3421 & 0.4952 & 0.4952 & 0.4976 \\
\hline
\end{tabular}

TABLE 17: The prominence of each criterion for example 3.

\begin{tabular}{lcccc}
\hline$m_{1}$ & $m_{2}$ & $m_{3}$ & $m_{4}$ & $m_{5}$ \\
\hline 1.2522 & 2.6842 & 0.9904 & 0.9904 & 0.9952 \\
\hline
\end{tabular}

TABLE 18: The discounting factor for example 3.

\begin{tabular}{lcccc}
\hline$m_{1}$ & $m_{2}$ & $m_{3}$ & $m_{4}$ & $m_{5}$ \\
\hline 0.7990 & 0.3726 & 1.010 & 1.010 & 1.005 \\
\hline
\end{tabular}

TABLE 19: Normalized discounting factor for example 3.

\begin{tabular}{lcccc}
\hline$m_{1}$ & $m_{2}$ & $m_{3}$ & $m_{4}$ & $m_{5}$ \\
\hline 0.1902 & 0.0896 & 0.2404 & 0.2404 & 0.2394 \\
\hline
\end{tabular}

4.3. Example 3. Assume that in a multisensor system, five sensors obtained five bodies of evidence, respectively. Suppose the real target is $A$, the five bodies of evidence are shown as follows:

$$
\begin{aligned}
& m_{1}(A)=0.5, m_{1}(B)=0.2, m_{1}(C)=0.3, \\
& m_{2}(B)=0.9, m_{2}(C)=0.1, \\
& m_{3}(A)=0.55, m_{3}(B)=0.1, m_{3}(A, C)=0.35, \\
& m_{4}(A)=0.55, m_{4}(B)=0.1, m_{4}(A, C)=0.35, \\
& m_{5}(A)=0.6, m_{5}(B)=0.1, m_{5}(A, C)=0.3 .
\end{aligned}
$$

The steps are shown in Tables 11-19, and the results calculated by different combination methods are shown in Table 20 and Figures 3 and 4, where we compare the proposed method with Dempster's combination rule, the simple averaging method proposed by Murphy [42], the weight averaging method by Deng et al. [43], and the method proposed by Song et al. [40]. 
TABLE 20: The results compared with other methods.

\begin{tabular}{|c|c|c|c|c|}
\hline & $m_{1}, m_{2}$ & $m_{1}, m_{2}, m_{3}$ & $m_{1}, m_{2}, \mathrm{~m}_{3}, m_{4}$ & $m_{1}, m_{2}, m_{3}, m_{4}, m_{5}$ \\
\hline \multirow{3}{*}{ Dempster's combination rule } & $m(A)=0$ & $m(A)=0$ & $m(A)=0$ & $m(A)=0$ \\
\hline & $m(B)=0.8571$ & $m(B)=0.6316$ & $m(B)=0.3288$ & $m(B)=0.1228$ \\
\hline & $m(C)=0.1429$ & $m(C)=0.3684$ & $m(C)=0.6712$ & $m(C)=0.8772$ \\
\hline \multirow{3}{*}{ Simple averaging method } & $m(A)=0.1543$ & $m(A)=0.3500$ & $m(A)=0.6027$ & $m(A)=0.7958$ \\
\hline & $m(B)=0.7469$ & $m(B)=0.5224$ & $m(B)=0.2627$ & $m(B)=0.0932$ \\
\hline & $m(C)=0.0988$ & $m(C)=0.1276$ & $m(C)=0.1346$ & $m(C)=0.1110$ \\
\hline \multirow{3}{*}{ Weighted averaging method } & $m(A)=0.1543$ & $m(A)=0.4861$ & $m(A)=0.7773$ & $m(A)=0.8909$ \\
\hline & $m(B)=0.7469$ & $m(B)=0.3481$ & $m(B)=0.0628$ & $m(B)=0.0086$ \\
\hline & $m(C)=0.0988$ & $m(C)=0.1657$ & $m(C)=0.1600$ & $m(C)=0.1005$ \\
\hline \multirow{3}{*}{ Song et al.'s method } & $m(A)=0.299$ & $m(A)=0.766$ & $m(A)=0.9250$ & $m(A)=0.9624$ \\
\hline & $m(B)=0.527$ & $m(B)=0.076$ & $m(B)=0.029$ & $m(B)=0.006$ \\
\hline & $m(C)=0.174$ & $m(C)=0.158$ & $m(C)=0.046$ & $m(C)=0.0032$ \\
\hline \multirow{3}{*}{ The proposed method } & $m(A)=0.8382$ & $m(A)=0.9334$ & $m(A)=0.9728$ & $m(A)=0.9890$ \\
\hline & $m(B)=0.0584$ & $m(B)=0.0162$ & $m(B)=0.0043$ & $m(B)=0.0011$ \\
\hline & $m(C)=0.1034$ & $m(C)=0.0504$ & $m(C)=0.0229$ & $m(C)=0.0091$ \\
\hline
\end{tabular}

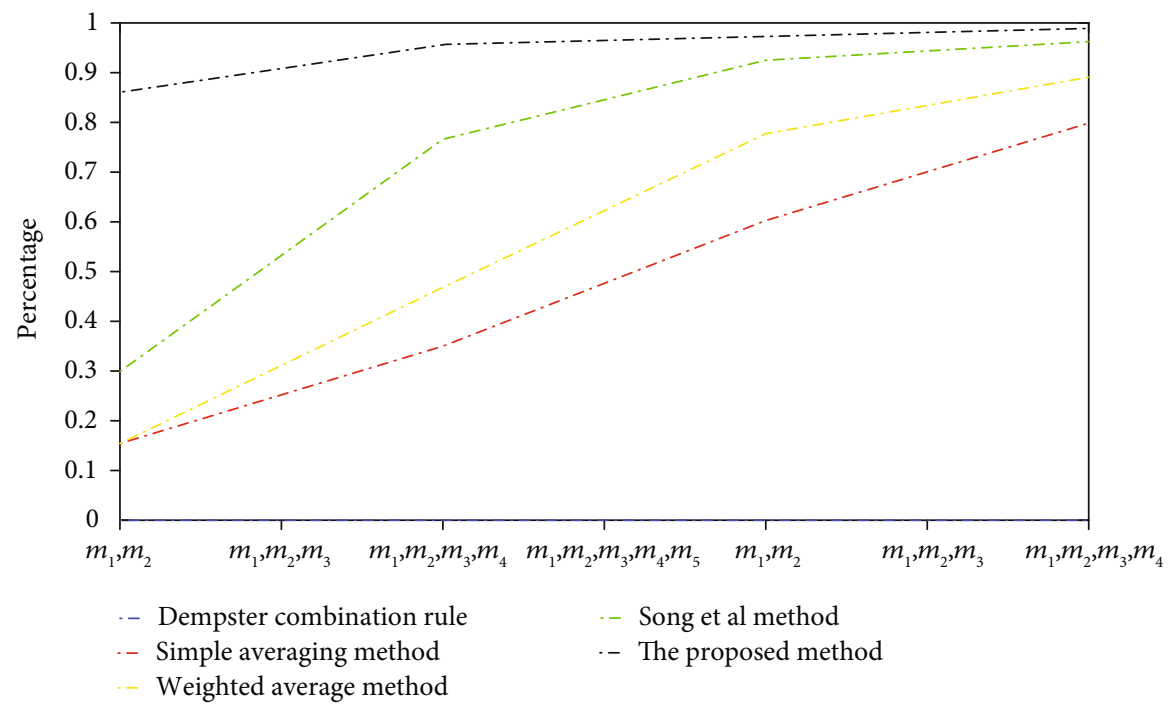

FIgURE 3: The line graph of probability of target $A$.

As shown in Table 20, due to the interfering BPA $m_{2}$, the results obtained by Dempster's combination rule show that although most of the evidence supports that the target is $A$, it ultimately provides most support for $C$, which is obviously unreasonable. The simple averaging method by Murphy [42], the weight averaging method by Deng et al. [43], and the method proposed by Song et al. [40] provide the reasonable results. It can be seen that the weighted average combination rule is effective in reducing the impact of extreme evidence on the results. However, compared with the methods above, the results obtained by the proposed new method reflect a stronger convergence, which more clearly supports the target $A$.

\section{Discussion and Advantages}

Dempster's combination rule regards all evidence as independent which makes it is a poor solution to manage the conflict between the various information sources. To some extent, averaging method [42] can solve this problem, but since it considers the importance of all evidence to be the same, the obtained result does not significantly reflect the convergence. Deng et al. consider the difference of evidence's importance and use evidence distance to calculate each evidence's weight to better handle the conflict management. The proposed method takes the new correlation coefficient proposed by Jiang as the measurement of the degree of 


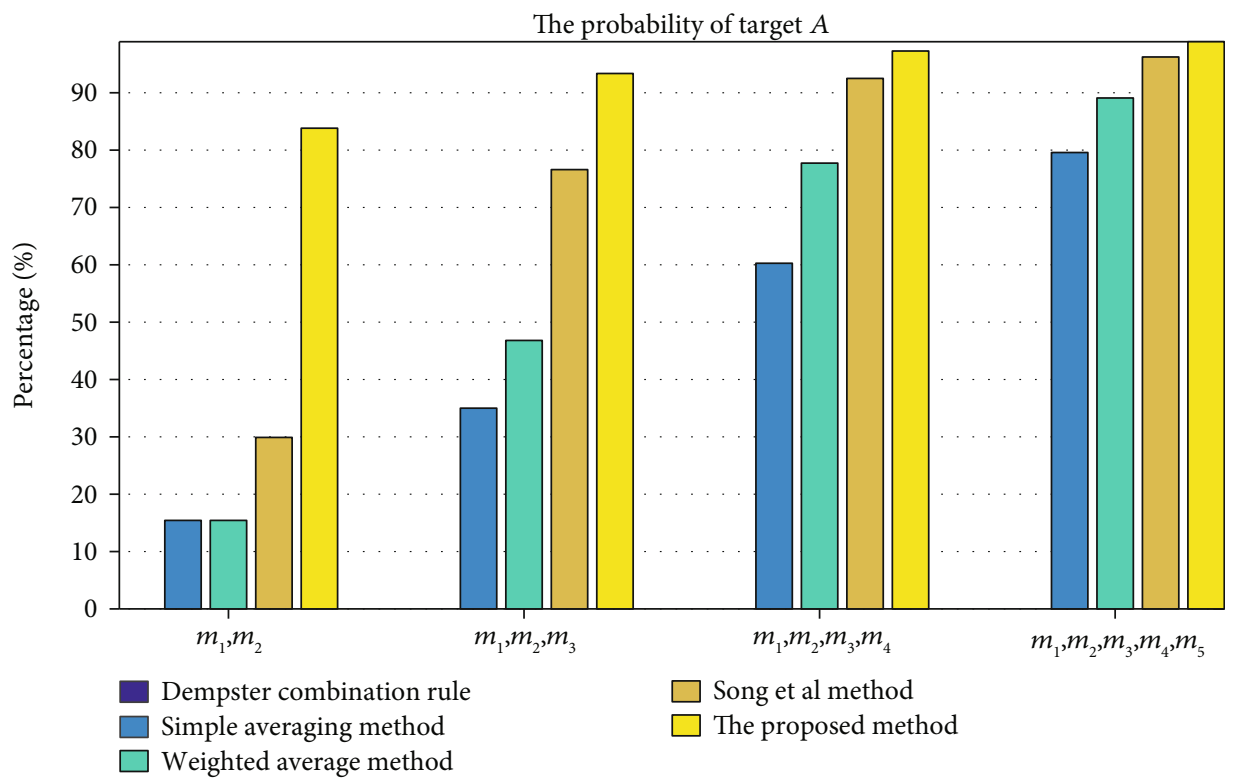

Figure 4: The histogram of probability of target $A$.

conflict. The new correlation coefficient takes both the noninteraction and the difference among focal elements into consideration which can more accurately reflect the conflict among evidence. Based on DEMATEL method, the proposed method considers the association relationship of the evidence and can efficiently handle conflict management with better performance of convergence. The reason why the DEMATEL method can handle the indirect effect is that in Step 3, normalized matrix continues to multiply and then add, this process is to superimpose the indirect effects of the elements. For the direct conflict is taken as the element of DEMATEL, the discounting factor obtained by DEMATEL is more reasonable and effective than other methods.

\section{Conclusion}

How to better handle conflict management to get a reasonable data fusion result is an open issue in evidence theory. Though the existing methods have been proved efficient to deal with conflict in some situations, the indirect conflict among evidence is neglected to some degree. This article proposed a method to combine belief function based on DEMATEL and correlation coefficient of BPA. In the case of considering indirect effects, the results obtained by DEMATEL clearly reflect the extent to which the element affects the entire system. Therefore, the discounting factor obtained by DEMATEL is more reasonable and effective than other methods. So, the numerical example shows that the results obtained by the proposed method have better performance compared with the other methods. The proposed method can be applied into group decision-making in the autonomous robotic systems or making information security risk control assessment in the future. Also, it has got significant technical and managerial implications. In a management system, even if there is a deviation in one component, the method can minimize the impact of the entire management system to make the right decision. The limita- tion is that the correlation coefficient cannot completely represent the direct conflict. And the proposed method cannot efficiently deal with data fusion with a large amount of data in real-time application systems.

\section{Data Availability}

The data used to support the findings of this study are included within the article.

\section{Conflicts of Interest}

The authors declare that they have no conflicts of interest.

\section{Acknowledgments}

This research was funded by the Chongqing Overseas Scholars Innovation Program (No. cx2018077).

\section{References}

[1] R. R. Yager, "Categorization in multi-criteria decision making," Information Sciences, vol. 460-461, pp. 416-423, 2018.

[2] F. Xiao, "EFMCDM: evidential fuzzy multicriteria decision making based on belief entropy," IEEE Transactions on Fuzzy Systems, p. 1, 2019.

[3] M. Zhou, X. Liu, and J. Yang, "Evidential reasoning approach for MADM based on incomplete interval value," Journal of Intelligent \& Fuzzy Systems, vol. 33, no. 6, pp. 3707-3721, 2017.

[4] Z. Cao, C. T. Lin, K. L. Lai et al., "Extraction of SSVEPs-based inherent fuzzy entropy using a wearable headband EEG in migraine patients," IEEE Transactions on Fuzzy Systems, p. 1, 2019.

[5] Y. Song and Y. Deng, "A new method to measure the divergence in evidential sensor data fusion," International Journal of Distributed Sensor Networks, vol. 15, no. 4, 2019. 
[6] H. Mo and Y. Deng, "An evaluation for sustainable mobility extended by D numbers," Technological and Economic Development of Economy, vol. 25, no. 5, pp. 802-819, 2019.

[7] X. Deng and W. Jiang, "Evaluating green supply chain management practices under fuzzy environment: a novel method based on D number theory," International Journal of Fuzzy Systems, vol. 21, no. 5, pp. 1389-1402, 2019.

[8] B. Liu and Y. Deng, "Risk evaluation in failure mode and effects analysis based on D numbers theory," International Journal of Computers Communications \& Control, vol. 14, no. 5, pp. 672-691, 2019.

[9] J. Zhao and Y. Deng, "Performer selection in human reliability analysis: D numbers approach," International Journal of Computers Communications \& Control, vol. 14, no. 3, pp. 437-452, 2019.

[10] W. Jiang, Y. Cao, and X. Deng, "A novel Z-network model based on Bayesian network and Z-number," IEEE Transactions on Fuzzy Systems, p. 1, 2019.

[11] B. Kang, P. Zhang, Z. Gao, G. Chhipi-Shrestha, K. Hewage, and R. Sadiq, "Environmental assessment under uncertainty using Dempster-Shafer theory and Z-numbers," Journal of Ambient Intelligence and Humanized Computing, pp. 1-20, 2019.

[12] H. Seiti, R. Tagipour, A. Hafezalkotob, and F. Asgari, "Maintenance strategy selection with risky evaluations using RAHP," Journal of Multi-Criteria Decision Analysis, vol. 24, no. 5-6, pp. 257-274, 2017.

[13] M. Zhou, X.-B. Liu, J.-B. Yang, Y.-W. Chen, and J. Wu, "Evidential reasoning approach with multiple kinds of attributes and entropy- based weight assignment," KnowledgeBased Systems, vol. 163, pp. 358-375, 2019.

[14] C. Fu, W. Chang, M. Xue, and S. Yang, "Multiple criteria group decision making with belief distributions and distributed preference relations," European Journal of Operational Research, vol. 273, no. 2, pp. 623-633, 2019.

[15] M. Zhou, X.-B. Liu, Y.-W. Chen, and J.-B. Yang, "Evidential reasoning rule for MADM with both weights and reliabilities in group decision making," Knowledge-Based Systems, vol. 143, pp. 142-161, 2018.

[16] Z.-g. Liu, Q. Pan, J. Dezert, and A. Martin, "Combination of classifiers with optimal weight based on evidential reasoning," IEEE Transactions on Fuzzy Systems, vol. 26, no. 3, pp. 12171230, 2018.

[17] D. Meng, S. Yang, Y. Zhang, and S.-P. Zhu, "Structural reliability analysis and uncertainties-based collaborative design and optimization of turbine blades using surrogate model," Fatigue \& Fracture of Engineering Materials \& Structures, vol. 42, no. 6, pp. 1219-1227, 2018.

[18] D. Meng, M. Liu, S. Yang, H. Zhang, and R. Ding, "A fluidstructure analysis approach and its application in the uncertainty-based multidisciplinary design and optimization for blades," Advances in Mechanical Engineering, vol. 10, no. 6, 2018.

[19] B. Kang and Y. Deng, “The maximum Deng entropy," IEEE Access, vol. 7, no. 1, pp. 120758-120765, 2019.

[20] Q. Wang, Y. Li, and X. Liu, "Analysis of feature fatigue EEG signals based on wavelet entropy," International Journal of Pattern Recognition and Artificial Intelligence, vol. 32, no. 8, article 1854023, 2018.

[21] Z. Cao and C.-T. Lin, "Inherent fuzzy entropy for the improvement of EEG complexity evaluation," IEEE Transactions on Fuzzy Systems, vol. 26, no. 2, pp. 1032-1035, 2018.
[22] R. R. Yager, “Generalized Dempster-Shafer structures," IEEE Transactions on Fuzzy Systems, vol. 27, no. 3, pp. 428-435, 2019.

[23] K. Chatterjee, D. Pamucar, and E. K. Zavadskas, "Evaluating the performance of suppliers based on using the R'AMATELMAIRCA method for green supply chain implementation in electronics industry," Journal of Cleaner Production, vol. 184, pp. 101-129, 2018.

[24] X. Su, L. Li, F. Shi, and H. Qian, "Research on the fusion of dependent evidence based on mutual information," IEEE Access, vol. 6, pp. 71839-71845, 2018.

[25] M. Tang, H. Liao, Z. Li, and Z. Xu, "Nature disaster risk evaluation with a group decision making method based on incomplete hesitant fuzzy linguistic preference relations," International Journal of Environmental Research and Public Health, vol. 15, no. 4, p. 751, 2018.

[26] X. Wang and Y. Song, "Uncertainty measure in evidence theory with its applications," Applied Intelligence, vol. 48, no. 7, pp. 1672-1688, 2018.

[27] Y. Pan, L. Zhang, Z. Li, and L. Ding, "Improved fuzzy Bayesian network-based risk analysis with interval-valued fuzzy sets and D-S evidence theory," IEEE Transactions on Fuzzy Systems, p. 1, 2019.

[28] Q. Liu, Y. Tian, and B. Kang, "Derive knowledge of Z-number from the perspective of Dempster-Shafer evidence theory," Engineering Applications of Artificial Intelligence, vol. 85, pp. 754-764, 2019.

[29] J. An, M. Hu, L. Fu, and J. Zhan, “A novel fuzzy approach for combining uncertain conflict evidences in the DempsterShafer theory," IEEE Access, vol. 7, pp. 7481-7501, 2019.

[30] X. Gao and Y. Deng, "The generalization negation of probability distribution and its application in target recognition based on sensor fusion," International Journal of Distributed Sensor Networks, vol. 15, no. 5, 2019.

[31] Y. Gong, X. Su, H. Qian, and N. Yang, "Research on fault diagnosis methods for the reactor coolant system of nuclear power plant based on D-S evidence theory," Annals of Nuclear Energy, vol. 112, pp. 395-399, 2018.

[32] C. Fu, W. Liu, and W. Chang, "Data-driven multiple criteria decision making for diagnosis of thyroid cancer," Annals of Operations Research, pp. 1-30, 2018.

[33] H. Seiti and A. Hafezalkotob, "Developing pessimisticoptimistic risk-based methods for multi-sensor fusion: An interval-valued evidence theory approach," Applied Soft Computing, vol. 72, pp. 609-623, 2018.

[34] J. Wang and F. Liu, "Temporal evidence combination method for multi-sensor target recognition based on DS theory and IFS," Journal of Systems Engineering and Electronics, vol. 28, no. 6, pp. 1114-1125, 2017.

[35] L. G. de Oliveira Silva and A. T. de Almeida-Filho, "A multicriteria approach for analysis of conflicts in evidence theory," Information Sciences, vol. 346-347, pp. 275-285, 2016.

[36] R. Sun and Y. Deng, "A new method to identify incomplete frame of discernment in evidence theory," IEEE Access, vol. 7, no. 1, pp. 15547-15555, 2019.

[37] X. Su, L. Li, H. Qian, S. Mahadevan, and Y. Deng, "A new rule to combine dependent bodies of evidence," Soft Computing, vol. 23, no. 20, pp. 9793-9799, 2019.

[38] A.-L. Jousselme and P. Maupin, "Distances in evidence theory: comprehensive survey and generalizations," International Journal of Approximate Reasoning, vol. 53, no. 2, pp. 118-145, 2012. 
[39] W. Liu, "Analyzing the degree of conflict among belief functions," Artificial Intelligence, vol. 170, no. 11, pp. 909924, 2006.

[40] Y. Song, X. Wang, J. Zhu, and L. Lei, "Sensor dynamic reliability evaluation based on evidence theory and intuitionistic fuzzy sets," Applied Intelligence, vol. 48, no. 11, article 1188, pp. 3950-3962, 2018.

[41] R. R. Yager, "On the fusion of non-independent belief structures," International Journal of General Systems, vol. 38, no. 5, pp. 505-531, 2009.

[42] C. K. Murphy, "Combining belief functions when evidence conflicts," Decision Support Systems, vol. 29, no. 1, pp. 1-9, 2000.

[43] D. Yong, S. WenKang, Z. ZhenFu, and L. Qi, "Combining belief functions based on distance of evidence," Decision Support Systems, vol. 38, no. 3, pp. 489-493, 2004.

[44] W. Jiang, C. Huang, and X. Deng, "A new probability transformation method based on a correlation coefficient of belief functions," International Journal of Intelligent Systems, vol. 34, no. 6, pp. 1337-1347, 2019.

[45] Z. Liu, Y. Liu, J. Dezert, and F. Cuzzolin, "Evidence combination based on credal belief redistribution for pattern classification," IEEE Transactions on Fuzzy Systems, p. 1, 2019.

[46] W. Jiang, "A correlation coefficient for belief functions," International Journal of Approximate Reasoning, vol. 103, no. 11, pp. 94-106, 2018.

[47] S. B. Tsai, J. Zhou, Y. Gao et al., "Combining FMEA with DEMATEL models to solve production process problems," PLoS One, vol. 12, no. 8, article e0183634, 2017.

[48] W. Zhang and Y. Deng, "Combining conflicting evidence using the DEMATEL method," Soft Computing, vol. 23, no. 17, pp. 8207-8216, 2019.

[49] F. Liu, G. Aiwu, V. Lukovac, and M. Vukic, "A multicriteria model for the selection of the transport service provider: a single valued neutrosophic dematel multicriteria model," Decision Making: Applications in Management and Engineering, vol. 1, no. 2, pp. 121-130, 2018.

[50] F. Feng, H. Fujita, M. I. Ali, R. R. Yager, and X. Liu, "Another view on generalized intuitionistic fuzzy soft sets and related multiattribute decision making methods," IEEE Transactions on Fuzzy Systems, vol. 27, no. 3, pp. 474-488, 2019.

[51] B. Wei, F. Xiao, and Y. Shi, "Synchronization in kuramoto oscillator networks with sampled-data updating law," IEEE Transactions on Cybernetics, pp. 1-9, 2019.

[52] Q. Wang and P. Lu, "Research on application of artificial intelligence in computer network technology," International Journal of Pattern Recognition and Artificial Intelligence, vol. 33, no. 5, article 1959015, 2019.

[53] F. Feng, M. Liang, H. Fujita, R. R. Yager, and X. Liu, "Lexicographic orders of intuitionistic fuzzy values and their relationships," Mathematics, vol. 7, no. 2, p. 166, 2019.

[54] D. Zhou, A. Al-Durra, K. Zhang, A. Ravey, and F. Gao, "A robust prognostic indicator for renewable energy technologies: a novel error correction grey prediction model," IEEE Transactions on Industrial Electronics, vol. 66, no. 12, pp. 9312-9325, 2019.

[55] J. Ma, W. Yu, P. Liang, C. Li, and J. Jiang, "Fusiongan: a generative adversarial network for infrared and visible image fusion," Information Fusion, vol. 48, pp. 11-26, 2019.
[56] X. Xu, H. Xu, C. Wen, J. Li, P. Hou, and J. Zhang, "A belief rule-based evidence updating method for industrial alarm system design," Control Engineering Practice, vol. 81, pp. 7384, 2018.

[57] Z. Liu, Q. Pan, J. Dezert, J.-W. Han, and Y. He, "Classifier fusion with contextual reliability evaluation," IEEE Transactions on Cybernetics, vol. 48, no. 5, pp. 1605-1618, 2018.

[58] X. Xu, J. Zheng, J.-b. Yang, D.-1. Xu, and Y.-w. Chen, "Data classification using evidence reasoning rule," Knowledge-Based Systems, vol. 116, pp. 144-151, 2017.

[59] R. R. Yager, "Entailment for measure based belief structures," Information Fusion, vol. 47, pp. 111-116, 2019.

[60] R. Sun and Y. Deng, "A new method to determine generalized basic probability assignment in the open world," IEEE Access, vol. 7, no. 1, pp. 52827-52835, 2019.

[61] X. Gao and Y. Deng, "The negation of basic probability assignment,” IEEE Access, vol. 7, no. 1, pp. 107006-107014, 2019.

[62] L. Fei and Y. Deng, "A new divergence measure for basic probability assignment and its applications in extremely uncertain environments," International Journal of Intelligent Systems, vol. 34, no. 4, pp. 584-600, 2019.

[63] Y. Dong, J. Zhang, Z. Li, Y. Hu, and Y. Deng, "Combination of evidential sensor reports with distance function and belief entropy in fault diagnosis," International Journal of Computers Communications \& Control, vol. 14, no. 3, pp. 329-343, 2019.

[64] L. A. Zadeh, "A simple view of the Dempster-Shafer theory of evidence and its implication for the rule of combination," $A I$ Magazine, vol. 7, no. 2, pp. 85-85, 1986.

[65] J.-B. Yang and D.-L. Xu, "Evidential reasoning rule for evidence combination," Artificial Intelligence, vol. 205, pp. 1-29, 2013.

[66] A.-L. Jousselme, D. Grenier, and É. Bossé, “A new distance between two bodies of evidence," Information Fusion, vol. 2, no. 2, pp. 91-101, 2001.

[67] Y. Liu and W. Jiang, "A New Distance Measure of Interval-Valued Intuitionistic Fuzzy Sets and Its Application in Decision Making," Soft Computing, pp. 1-17, 2019.

[68] Y. Wang, K. Zhang, and Y. Deng, "Base belief function: an efficient method of conflict management," Journal of Ambient Intelligence and Humanized Computing, vol. 10, no. 9, pp. 3427-3437, 2019.

[69] S. G. Hofmann, J. Curtiss, and R. J. McNally, "A complex network perspective on clinical science," Perspectives on Psychological Science, vol. 11, no. 5, pp. 597-605, 2016.

[70] B. Wei, F. Xiao, and Y. Shi, "Fully distributed synchronization of dynamic networked systems with adaptive nonlinear couplings," IEEE Transactions on Cybernetics, pp. 1-9, 2019.

[71] F. Rundo, S. Conoci, A. Ortis, and S. Battiato, "An advanced bio-inspired photoplethysmography (PPG) and ECG pattern recognition system for medical assessment," Sensors, vol. 18, no. 2, p. 405, 2018.

[72] H. Yang, Y. Liu, Q. Wan, and Y. Deng, "A bio-inspired optimal network division method," Physica A: Statistical Mechanics and its Applications, vol. 527, article 121259, 2019.

[73] H. Yang, Y. Deng, and R. Mayne, "A bio-inspired network design method for intelligent transportation," International Journal of Unconventional Computing, vol. 14, no. 3,4, pp. 199-215, 2019. 
[74] H. Yang, Y. Deng, and J. Jones, "Network division method based on cellular growth and physarum-inspired network adaptation," International Journal of Unconventional Computing, vol. 13, no. 6, pp. 477-491, 2018.

[75] W.-W. Wu and Y.-T. Lee, "Developing global managers' competencies using the fuzzy DEMATEL method," Expert Systems with Applications, vol. 32, no. 2, pp. 499-507, 2007. 


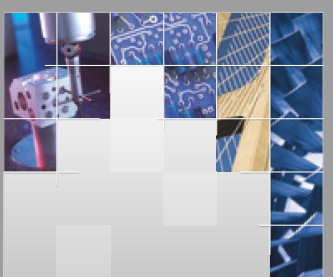

\section{Enfincering}
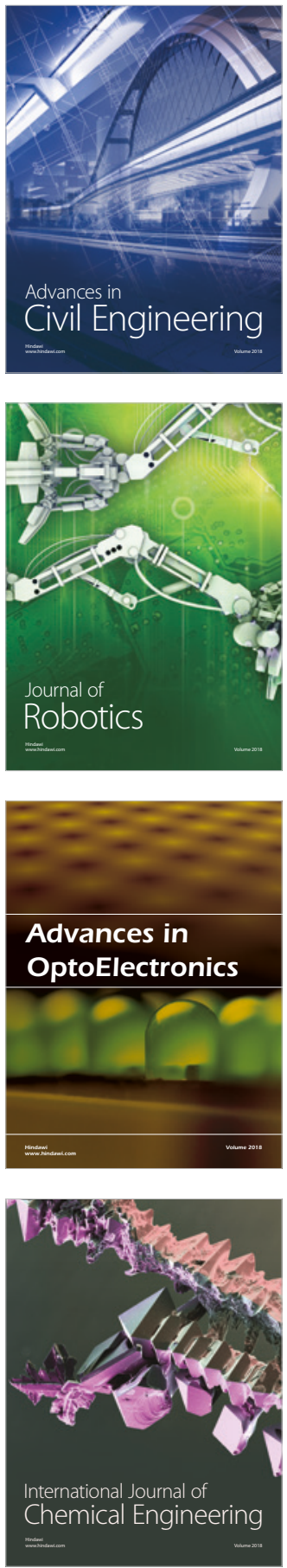

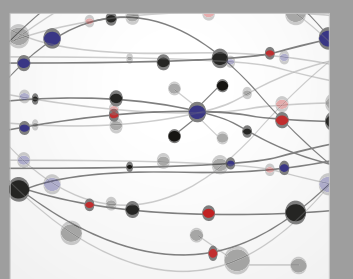

\section{Rotating \\ Machinery}

The Scientific World Journal

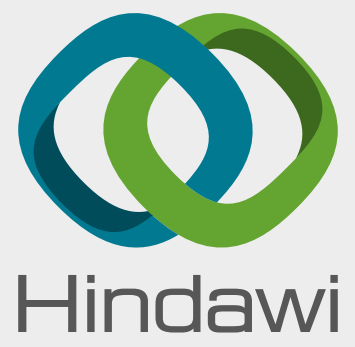

Submit your manuscripts at

www.hindawi.com
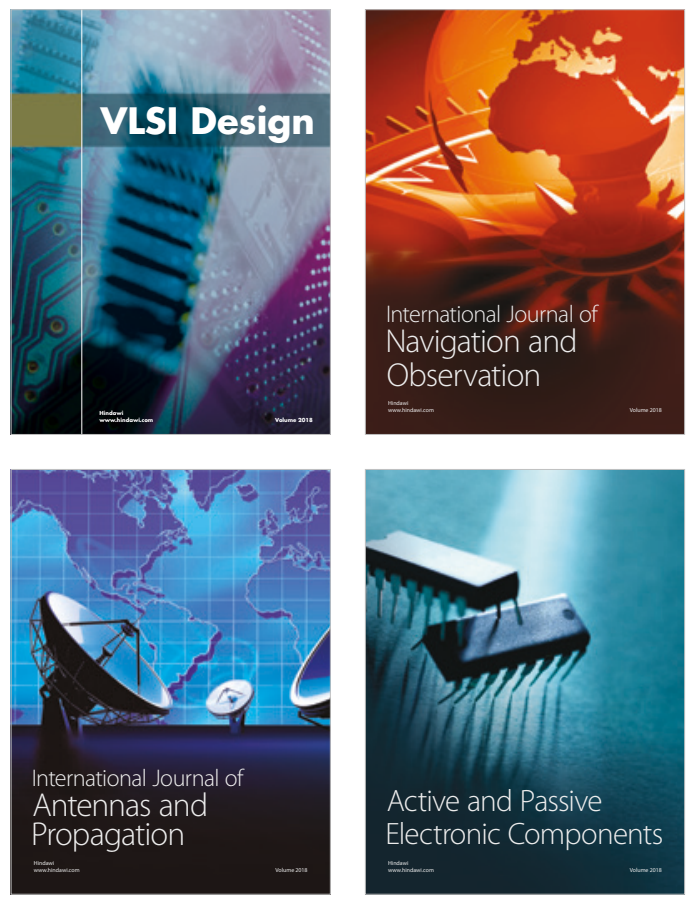
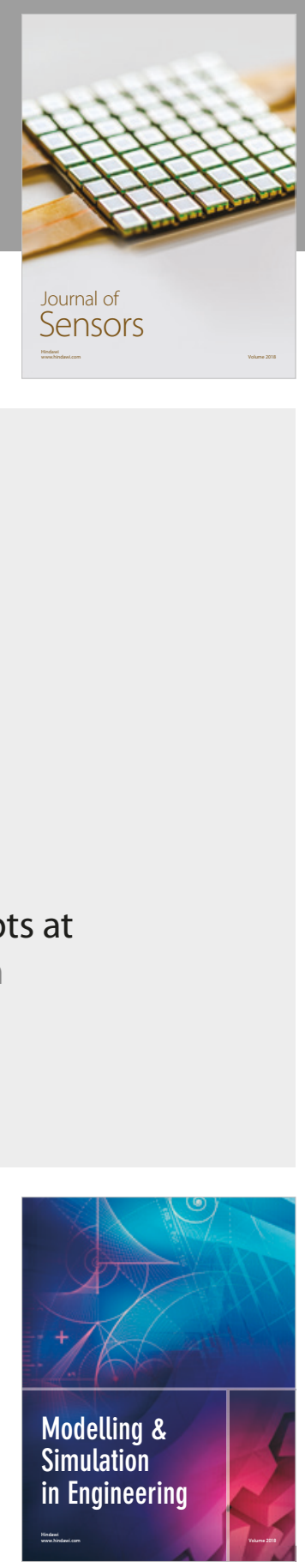

\section{Advances \\ Multimedia}
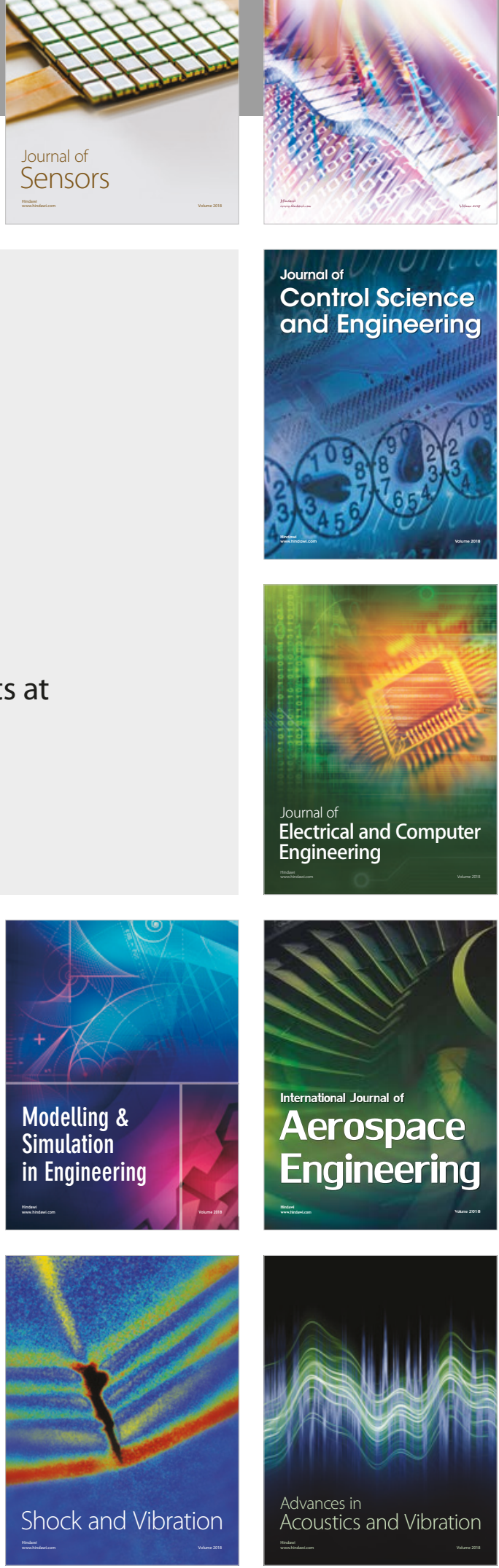